\title{
Marvel-lous Masked Men. Doppelidentitäten in Superheldenfilmen
}

\author{
von Holzen, Aleta-Amirée
}

DOI: https://doi.org/10.1515/9783110276732.187

Posted at the Zurich Open Repository and Archive, University of Zurich

ZORA URL: https://doi.org/10.5167/uzh-63540

Book Section

Published Version

Originally published at:

von Holzen, Aleta-Amirée (2012). Marvel-lous Masked Men. Doppelidentitäten in Superheldenfilmen. In: Schmeink, Lars; Müller, Hans-Harald. Fremde Welten. Wege und Räume der Fantastik im 21. Jahrhundert. Berlin, Boston: de Gruyter, 187-202.

DOI: https://doi.org/10.1515/9783110276732.187 


\title{
Marvel-lous Masked Men \\ Doppelidentitäten in Superheldenfilmen ${ }^{1}$
}

\author{
ALETA-AMIRÉE VON HOLZEN
}

Marvel-lous Masked Men. Double Identities in Super Hero Films

This article focuses on recent film adaptations of Marvel comics' superheroes SpiderMan (2002, 2004, 2007), Daredevil (2003) and Iron Man (2008). By wearing a mask while acting as heroes in the public, these characters establish a double identity and thereby embody the figure of the 'masked hero', which is considered a narrative prototype. In a first part, the role of the superpowers as precondition for the heroism of the characters is illuminated, in relation to their classic comic versions. The double identity (which is not purely a superhero trait) is considered to be a vital part of the hero figuration leading up to typical plot elements in the narrative. How these unfold around matters of masquerade is explored in this article.

Powers and mask provide their bearer with the means of an identity enhancement. Thus, this paper will further argue that this mask-based double identity not only dwells in questions of identity but mirrors modern identity concepts. As portrayed in these movies, the three superheroes combine in their identity a constant change (switching from heroic to 'secret' identity) as well as steadiness, since the double-identity, no matter how seriously questioned in the story, eventually remains as first established.

Seit Stan Lee 1962, 1963 und 1964 Spider-Man, Iron Man und Daredevil ersonnen hat, haben die drei Superhelden unzählige Abenteuer in gezeichneter Form bestanden, hauptsächlich in Comic-Heften, aber auch in Trickfilmserien. ${ }^{2}$ Doch während ihre Konkurrenten aus dem Comic-Universum von DC, allen voran Superman und Batman, den Sprung auf die Kinoleinwand schon im 20. Jahrhundert geschafft haben, schien für die Marvel-Superhelden ein Durchbruch im Kino-Realspielfilm aus verschiedenen

1 Für den englischen Teil des Titels danke ich Petra Schrackmann. Dieser Beitrag steht in Zusammenhang mit dem Dissertationsprojekt der Verfasserin, das sich jedoch nicht auf Filme fokussieren wird. Das Dissertationsprojekt wird für zwei Jahre als Teil des Projekts „Übergänge und Entgrenzungen. Welt, Wissen und Identität in fantastischer (Kinder- und Jugend-)Literatur und ihren Verfilmungen“ unterstützt durch den SNF (Schweizerischer Nationalfonds).

2 1978-79 gab es zudem eine 15-teilige Live-Action-Fernsehserie mit Spider-Man (Booker 110). 
Gründen lange eher unwahrscheinlich (vgl. Booker). Erst nachdem der Erfolg des Films X-Men im Jahr 2000 das Vertrauen in die Möglichkeiten der fotorealistischen Computeranimation gestärkt und den Weg beim Publikum gebahnt hatte, gelangten die drei berühmtesten maskierten Einzelgänger aus dem Hause Marvel zu ihren ersten Umsetzungen auf der großen Leinwand. Die Titel der Kino-Adaptionen sind dabei ebenso zweckmäßig wie simpel: Spider-Man (2002), Spider-Man 2 (2004), Spider-Man 3 (2007)3, Daredevil (2003) sowie Iron Man (2008) und Iron Man 2 (2010). Den ersten fünf dieser sechs Filme widmet sich dieser Beitrag, wobei das Hauptaugenmerk Spider-Man und Daredevil gilt.

Im Mittelpunkt steht dabei die Doppelidentität des maskierten Superhelden, die besondere ,Identitätsform' der Protagonisten, deren Thematisierung in diesen Filmen unter die Lupe genommen wird. Sie wird darüber hinaus in Bezug zu (post-)modernen Identitätskonzeptionen gesetzt. Da es sich bei diesen Filmen um Adaptionen handelt, wird immer wieder auf die klassischen ${ }^{4}$ Comicversionen dieser Charaktere verwiesen, ein Vergleich zwischen Comics und Film ist jedoch nicht das hauptsächliche Ziel dieses Beitrags.

Analog zu den klassischen Comics beginnen die Geschichten von Spider-Man, Iron Man und Daredevil auch bei ihrer Neuauflage im Film mit der sogenannten Origin Story, die erklärt, wie aus dem Protagonisten ein Superheld wird. Das Geschehen wurde dabei konsequenterweise in die Gegenwart versetzt, da Superheldencomics im Allgemeinen in der jeweiligen Gegenwart ihrer Entstehungszeit situiert sind. Jede der in diesen filmischen Adaptionen erzählten Origin Storys lässt sich wie folgt zusammenfassen: Nach dem Erhalt oder der Erlangung bzw. Erschaffung übernatürlicher Kräfte macht der Protagonist eine Verlusterfahrung, wenn eine väterliche Figur gewaltsam stirbt. Das dabei erlittene Ohnmachtsgefühl veranlasst ihn dazu, seine Superfähigkeiten für die Allgemeinheit einzusetzen, besonders aber gegen Superschurken, die bei Superhelden selten lange auf sich warten lassen. Die Superschurken verfügen in der Regel ähnlich wie die Helden über eine Origin Story, und es ist inzwischen eine Binsenweisheit, dass sie häufig eine ins Negative gewendete Spiegelung des Helden, einen bösen Zwilling oder, wie Tyree formuliert, ,an identical - but evil - version of himself" (28), darstellen (sollen). Obwohl sich diesbe-

3 Ein geplanter vierter Teil der Spider-Man-Reihe wurde inzwischen zu einem sogenannten ,Reboot', also einer eigenständigen, nicht mit der bisherigen Trilogie verknüpften Umsetzung, umgeschrieben; dieser Film soll 2012 in die Kinos kommen (vgl. dazu <www.imdb.com/ title/tt0948470>, letzter Abruf 12. 01. 2012).

4 Als solche verstanden werden hier insbesondere die ersten Jahrgänge jener Comic-Hefte, in denen die drei Charaktere namengebend oder fester Bestandteil waren (das sind Amazing Spider-Man, Daredevil und Tales of Suspense des Marvel-Verlags), spätere Neuerzählungen und Sonderreihen wurden hier nicht berücksichtigt. 
züglich von einer Doppelung des Helden (und einem ,maskierten Schurken?) sprechen ließe, beschränkt sich dieser Beitrag auf die den Heldenfiguren eigenen Doppelidentitäten.

Spider-Man, Iron Man und Daredevil sind nicht nur Superhelden - ihre Gestalten entsprechen auch dem maskierten Helden. ${ }^{5}$ Diesen verstehe ich als Figurentypus, der das Grundmodell für diverse populäre Heldengestalten darstellt und der nicht notwendigerweise in fantastischen Genres auftreten muss (wohl prominentestes nicht-fantastisches Beispiel ist Johnston McCulleys Zorro). Helden und Heldinnen ${ }^{6}$ nach diesem Modell erschaffen sich mit Hilfe einer Maske, und oft eines Kostüms, eine zweite Identität ${ }^{7}$, in der sie ihre Heldentaten vollbringen, während sie ihre heldischen Fähigkeiten in ihrem sozialen Umfeld verbergen und so gleichwohl ihre zivile Identität (im Superhelden-Fanjargon als Secret Identity bekannt) aufrechterhalten. Die Superhelden stellen weitaus die zahlreichsten Manifestationen des maskierten Helden. Bei ihnen wird die Doppelidentität mit Superkräften ${ }^{8}$ kombiniert. Im Folgenden wird zunächst geklärt, welche Rolle die fantastischen Elemente für die Doppelidentität des Helden - auch im Vergleich zu den Comics - spielen.

\section{Fantastische Fähigkeiten als Voraussetzung für das Heldendasein}

Für Superhelden sind ihre fantastischen Fähigkeiten die Voraussetzung, um als Held agieren zu können; diese sind denn auch ein Definitionskriterium des Superhelden (Coogan 30-61). Der Erhalt der Kräfte durch den schicksalhaften Spinnenbiss (Peter Parker/Spider-Man), den Unfall mit einem Chemielaster (Matt Murdock/Daredevil) oder die Fertigung einer Superrüstung (Tony Stark/Iron Man) bedeutet für die Protagonisten den Einbruch des Fantastischen in ihr bis anhin nicht-fantastisches Dasein. Sowohl für die Protagonisten als auch für die Rezipierenden manifestiert sich in den Superkräften das Fantastische als Novum bzw. mehrere Nova - wunderbare Elemente, die in der Realität des Publikums nicht existieren,

5 Superhelden sind nicht gleichbedeutend mit maskierten Helden; es gibt durchaus Superhelden wie die Fantastic Four und viele der X-Men, die keine Maske und keine Doppelidentität haben.

6 Im Folgenden wird nur die männliche Bezeichnung verwendet; selbstverständlich bezieht sich der Begriff des maskierten Helden ebenso auf weibliche Figuren, die diese Eigenschaften aufweisen, die aber leider stark in der Unterzahl sind.

7 Die Begriffe Identität, Ich und Selbst werden in diesem Beitrag synonym verwendet.

8 Als Superhelden gelten auch Figuren wie Batman, die keine körperliche Superkraft haben, sondern sich mit fantastischen Gadgets, also technischen Mitteln (z. B. Batcar, Batarang), behelfen (Coogan 41). Dazu zählt auch Tony Stark alias Iron Man mit seiner transhumanistisch anmutenden Superrüstung. 
also realitätsinkompatibel sind (Spiegel 42f.; unter Berufung auf Darko Suvin). Für den „fiktional-ästhetischen Modus“ (ebd. 39) der Science Fiction, zu dem Superheldengeschichten gemeinhin gezählt werden, ist charakteristisch, dass stets „eine prinzipielle Vereinbarkeit ihrer Nova mit dem aktuellen Stand der Wissenschaft" (ebd. 48) suggeriert wird.

Dies zeigt sich auch in den hier betrachteten Filmen: So wird Peter Parker im Film, anders als bei seinem ersten Auftritt im Comic, nicht von einer radioaktiven, sondern von einer genmanipulierten Spinne gebissen obwohl der Film neben den futuristischen Gleitern des Green Goblin, des Antagonisten, eine von einer unspezifizierten, idealisierten Nostalgie geprägte ,Alltagswelt ${ }^{\star}$ präsentiert, in welcher etwa Handys nicht zu existieren scheinen (vgl. Koh 736-39). Ebenso beruht Tony Starks geniale Rüstung, die sein Überleben nach einer unheilbaren Verwundung sichert, auf Reaktoren- statt Generatorentechnik. Da die Filme ihre Nova stark auf Superkräfte zentrieren, lässt sich behaupten, dass das Fantastische hier stark figurgebunden ist. Alle auf menschlichen Erfindungen beruhenden Nova führen in den Filmen zur fantastischen Veränderung eines Menschen oder unterstützen diese zumindest. Sogar der außerirdische Symbiont in SpiderMan 3 entfaltet seine Wirkung erst auf einem menschlichen Träger. Stellenweise bieten die Filme kleine, aber nicht unbedeutende Verschiebungen in der Ausgestaltung der Fähigkeiten. So wird Peter Parker zwar als Wissenschaftsgenie beschrieben, aber nie als solches, bei der Arbeit' gezeigt. Insbesondere ist Spider-Mans Netzflüssigkeit nicht das geniale Produkt seines Erfindergeistes, sondern eine der Wirkungen, die der Spinnenbiss auf seinen Körper hatte. Im Film Daredevil bleiben die Superkräfte des Helden das einzige Novum: Zum einen fehlt der Wissenschaftsnimbus, der Spider-Man und Iron Man umgibt, zum andern gibt es keine Antagonistenfiguren, die durch fantastische Elemente erklärte Superkräfte besitzen. ${ }^{9}$

Der Erhalt ihrer Kräfte bedeutet für Tony Stark, Peter Parker und Matt Murdock eine Erweiterung der Welt. Mit seinen Fähigkeiten kann sich der Held in der urbanen Situierung besondere Räume erschließen. Für Iron Man mit seinen Flugstabilisatoren ist es der freie Himmel, für Spider-Man und Daredevil sind es die Dächer New Yorks und die Häuserschluchten zwischen den Wolkenkratzern. Hier eröffnet sich ein dem Helden eigener Aktionsraum: Wo der Aufenthalt für Normalsterbliche

9 Der Kingpin als Drahtzieher und der Auftragskiller Bullseye als dessen Mittelsmann sind in Daredevil zwar überhöhte Gestalten, insbesondere die Agilität und Zielsicherheit Bullseyes bleiben aber unerklärt. (In den Comics zählt Bullseye ebenfalls zu jenen Figuren, die keine ,eigentlichen' Superkräfte haben, sondern ihre Talente durch Training zu übermenschlichen Ausmaßen geformt haben.) Elektras Kampfkraft wird explizit mit jahrelangem Training begründet. Allerdings werden auch Matt Murdocks Kräfte etwas zurückgenommen: Im Film ist er auf Blindenschrift angewiesen, derweil er in den Comics mit seinen hochsensitiven Fingerspitzen die Druckerschwärze auf dem Papier erfühlen kann. 
ohne Hilfsmittel tödliche Folgen hätte, kann der Held seine ganze Agilität unter Beweis stellen. Peaslee betont die Bedeutung der Großstadt als Schauplatz der Geschichte und ihre sozialen Implikationen für Spider-Man 1, seine Ausführungen können jedoch auch für die Spider-Man-Sequels, Daredevil und teilweise Iron Man Gültigkeit beanspruchen:

the setting $[\ldots]$ is one of extreme urbanity. $[\ldots$ T] he geography of skyscrapers, subways, sewers, and compartmentalizing spaces play a constitutive role in not only the mood but also the action of the story. [...] Only when he [Peter Parker] is Spider Man [sic], is he capable of transcending the maze of urban structure, as he leaps from building to building with the aid of his webs. (39)

Ihre spezielle Fortbewegungsart ist für Spider-Man und Daredevil fester Bestandteil ihrer Ikonographie geworden; im mühelosen Schwingen zwischen den Hochhäusern wird die Freude des Helden am Heldsein, an seinen Fähigkeiten, sichtbar. Matt Murdock/Daredevil, dessen Chemielaster-Unfall im Ausgleich zu Blindheit zu überscharfen restlichen Sinnen führte, formuliert dies explizit: „An acute sense of touch gave me both strength and balance until the city itself became my playground" (00:12:20; meine Hervorhebung).

Wie stark die Fähigkeit, durch Körperbeherrschung ungewohnte Räume durchqueren zu können, mit der Vorstellung des Superhelden verbunden ist, zeigt die komische Szene in Spider-Man 2, in der Spider-Man plötzlich seine Kräfte verliert und den Lift nehmen muss: Sein Mitfahrer hält ihn für irgendwen in einem Spider-Man-Kostüm, kommt aber nicht auf die Idee, dass das Original vor ihm steht. Freilich werden solche Räume meist schon bald auch von Superschurken beansprucht, etwa dem Green Goblin auf seinem Gleiter (Spider-Man 1) oder Doctor Octopus mit seinen überdimensionalen Metallarmen (Spider-Man 2). Durch die Superkräfte wird der im Spektrum der Stadt sonst eng begrenzte Freiraum für den Helden nahezu maximiert.

Im Vergleich mit den Comics wirkt die räumliche Erweiterung in den hier besprochenen Filmen allerdings ziemlich eingeschränkt. Für Iron Man und seine flugfähige Rüstung wäre theoretisch der gesamte Globus ein potentielles Aktionsfeld - der Showdown mit dem Iron Monger findet jedoch vor seiner Haustür statt. In den Comics regelmäßig erscheinende Elemente wie versunkene Welten, abgelegene Kleinreiche mit seltsamer Bevölkerung oder Eroberer aus dem Weltall übernehmen die Adaptionen sehr zögerlich. Erst in Spider-Man 3 gibt es mit dem außerirdischen Symbionten einen fantastischen Gegner, der nicht von der Erde stammt. Dies ist wohl im Anspruch der Filme begründet, die fantastischen Elemente so real wie möglich wirken zu lassen und die dargestellte Welt so nah wie möglich an jener des Kinopublikums zu orientieren (vgl. Booker 111). 


\section{Identitätserweiterung mittels Maske}

Diesen entgrenzten Raum nutzen die hier betrachteten drei Superhelden gewöhnlich jedoch nur mit einer besonderen Bedingung: Zwar sind seine fantastischen Fähigkeiten die Voraussetzung für das Heldentum des jeweiligen Protagonisten, dieser erlegt sich jedoch auf, seine Kräfte in der Öffentlichkeit nur dann aktiv einzusetzen, wenn er maskiert ist, d. h. wenn seine zivile Existenz aufgrund seiner äußeren Erscheinung nicht identifizierbar ist. Das Fantastische bedeutet daher nicht nur eine Erweiterung der Welt, sondern auch eine Erweiterung der Identität, die jedoch nur durch das Tragen einer Maske öffentlich sichtbar wird, der Maske, die seine Doppelidentität ermöglicht und zu seinem Markenzeichen wird.

Auffallenderweise vermeiden es die Filme - anders als die klassischen Comics -, einen konkreten Grund für die Maskierung zu nennen; die Maskierung und damit die Doppelidentität wird so als gegebener Bestandteil des Heldenlebens präsentiert, was hauptsächlich damit zusammenhängen dürfte, dass das ,Schema Superheld' als bekannt vorausgesetzt wird.10 Allerdings hebt ein farbintensives Kostüm bzw. eine Rüstung deutlich von der Allgemeinheit ab, derweil die Superkräfte in der zivilen Identität problemlos verborgen werden können.

Alle drei Superhelden erwähnen in den Filmen mindestens einmal mehr oder weniger explizit, dass der Grund, weshalb sie sich zum Heldenleben berufen bzw. verpflichtet fühlen, schlicht darin liegt, weil sie dazu in der Lage sind. Wenn Matt Murdock auf die Frage, warum gerade er derjenige ist, der ,etwas unternehmen“ muss, knapp antwortet: „Because I can. Because I'm not afraid“" (Daredevil 00:30:23), bezieht er sich in erster Linie klar auf sein übermenschliches Können, doch stellt sich die Frage, ob dieses ,Können' nicht auch von seiner Maske - und damit der Doppelidentität - abhängt.

Bei Iron Man wird die Maske als Visier der Rüstung sogar selbst zum fantastischen Element. Die Filmversion von Iron Man, ein gekonntes Spiel mit den Genrekonventionen, ist jedoch ein Spezialfall, da der Film die typische Doppelidentität - die in den Comics allen drei Helden gemeinsam ist -, verwirft. ${ }^{11}$ Ein mögliches Doppelleben wird zwar angesprochen, aber konsequent sabotiert, wenn Tony Stark schon beim ersten richtigen Ausflug als Iron Man erst seinen besten Freund Rhodey einweihen muss, um

10 Ein Indiz hierfür ist eine Anspielung auf den ersten aller Superhelden, wenn Tante May Peter fürsorglich ermahnt: „You do too much. [...] You're not Superman, you know“ (Spider-Man 01:33:45).

11 Fingeroth (59f.) weist darauf hin, dass die Secret Identity in den Comics im Lauf der Jahre immer mehr an Bedeutung verloren habe, aber in den Verfilmungen als wichtiges Thema vorhanden sei. 
nicht von dessen Kampfflugzeugen abgeschossen zu werden, und bei der Rückkehr dann von seiner Sekretärin Pepper Potts ertappt wird. Als Tony Stark die Doppelidentität am Schluss des Films tatsächlich etablieren soll, gesteht er vor versammelter Presse: „I am Iron Man“ (Iron Man 01:52:20). ${ }^{12}$

Ein entsprechendes Bekenntnis ist auch der Schlusssatz in Spider-Man, und ein vergleichbares Statement macht Matt Murdock in Daredevil (,This is who I am“; 01:17:30), bevor er sich noch einmal aufrappelt, um sich dem auf ihn angesetzten Killer Bullseye zu stellen. Diese Deklarationen deuten eine Tendenz an, die den Geschichten um maskierte Helden inhärent ist: Die besondere Konzeption der Heldenfigur fordert die Thematisierung von Identität heraus und bietet die Möglichkeit einer narrativen Problematisierung.

Die Doppelidentität des maskierten Helden, die äußerliche Trennung in zwei Identitäten, die nur alternierend auftreten können, gewinnt ihren erzählerischen Reiz insbesondere dadurch, dass das zivile und das HeldenIch in Kontrast stehen: Der maskierte Held spielt in seiner zivilen Identität den Unscheinbaren oder Helden-Untauglichen, im Fall von Daredevil sogar den Behinderten. ${ }^{13}$

Die Kontrastierung der beiden Helden-Ichs geschieht in den Geschichten unter anderem durch die unterschiedliche Bewertung, die das Umfeld des Protagonisten seinen beiden Ichs zukommen lässt, was sowohl komische als auch tragische Effekte haben kann. Eine dramatisch zugespitzte Repräsentation dieses Kontrasts verkörpert in der Regel eine Frauenfigur als love interest des Helden. Dies ist auch in den Filmhandlungen der Fall: Spider-Man 1 referiert die ,romantische“ Variante, wenn Mary Jane für Spider-Man schwärmt, aber Peter als Partner nicht bzw. noch nicht in Betracht zieht. Daredevil präsentiert indes eine Variante der extremen Polarisation: Elektra verliebt sich in Matt Murdock, dessen heldisches Alter Ego Daredevil dagegen soll von ihrer Hand sterben, da sie den gewaltsamen Tod ihres Vaters an dessen vermeintlichem Mörder rächen will. Doch nicht nur Frauenfiguren können die Gegensätzlichkeit der beiden äußerlichen Helden-Ichs herausstreichen, meist gibt es mehrere Figuren, die diesem Zweck dienen: In der Spider-Man-Trilogie möchte Peters bester Freund Harry Osborne Spider-Man tot sehen, da er ihn ebenfalls für den Tod seines Vaters Norman, der als Superbösewicht Green Goblin sein Unwesen getrieben hatte, verantwortlich macht. Zeitungsverleger J. Jonah Jameson sorgt für (eher komische) Kontraste, wenn er eine mediale

12 Die Aufhebung der Doppelidentität erklärt den Ausschluss von Iron Man 2 (2010, Regie: Jon Favreau) aus dem Korpus dieses Beitrags, und aus diesem Grund beziehen sich die folgenden Ausführungen nur noch sporadisch auf Iron Man.

13 Der blinde Matt Murdock hat zwar eine Behinderung, doch kann er diese derart kompensieren, dass er wie ein Nichtbehinderter auftreten könnte - dennoch verschweigt er seinem Umfeld seine kompensatorischen Fähigkeiten weitgehend. 
Hetzkampagne gegen Spider-Man veranstaltet, ohne zu wissen, dass dieser ihm als Pressefotograf Peter Parker selbst die Fotos dazu liefert.

Die mittels der Maske für den Blick von außen scheinbar getrennten Identitäten des Helden sind für diesen selbst und die Rezipierenden, die ja in das Geheimnis der Doppelidentität eingeweiht sind, durch ein Netz von Bezügen verbunden. In den hier betrachteten Filmen stoßen die Heldenfiguren auffallend oft mit ihrer zivilen Identität auf ihre nächste Aufgabe: Iron Man hat von Anfang an vor allem den Zweck, die Verfehlungen Tony Starks als Waffenproduzent zu korrigieren, Matt Murdock verfolgt als Daredevil nachts jene Unholde, die er tagsüber als Anwalt ungestraft davonkommen sah. ${ }^{14}$ Peter Parker ist praktischerweise meist als Fotograf vor Ort, wenn ein neuer Superschurken-Charakter seinen ersten Angriff auf die Stadt und ihre Bewohner startet. Auch in eher unspektakuläreren Situationen nimmt Peter gelegentlich sein Helden-Ich zu Hilfe, beispielsweise zu Beginn von Spider-Man 2: Zur Erfüllung seines Pizzakurier-Jobs verwandelt er sich in Spider-Man, weil er aber unterwegs Kinder retten muss, kommt er trotzdem zu spät an seine Zieldestination und verliert so auch diesen Job. Das heldische Alter Ego kann hier das Problem nicht lösen. Typischerweise werden Bedrohungen, die den Einsatz des Helden erfordern, ausgerechnet dann akut, wenn es für das soziale Leben des zivilen Ichs äußerst ungünstig ist, z. B. bei einem wichtigen Gespräch mit der Geliebten.

\section{Vom,wahren' Ich zum Protean Self}

Nach Tseëlon (3) bedeutet das Tragen einer Maske stets die Infragestellung der Identität. Sie führt weiter aus, dass es zwei mögliche Herangehensweisen gibt, um die Maskerade in Bezug auf die Identität zu verorten, abhängig davon, welcher der beiden Identitäts-Grundvorstellungen des 20. Jahrhunderts man folgen möchte, ob man Identität als eine feste Einheit versteht oder die Vorstellung einer grundsätzlich multiplen Identität vorzieht (ebd. 4). Geht man von Identität als einer festen Einheit aus, würde die Maske ein authentisches Ich verbergen. Hieraus erklärt sich die tendenziell negative Konnotation der Maske als Schein und Betrug, deren geistesgeschichtliche Entstehung Weihe umfassend erläutert. In den hier betrachteten Filmen referieren Jonah Jameson (Spider-Man) und der King-

14 Zum „A lawyer during the day [...] and then judge and jury at night“-Motto in Daredevil, das sein Beichtvater Matt einmal vorhält (00:30:13) vgl. Rauscher (67). In Daredevil zieht auch der Journalist Ben Urich die Verbindung zwischen den beiden Identitäten des Helden; tatsächlich versucht er zu beweisen, dass das Gerücht um Daredevil wahr ist und sich hinter dessen Maske Matt verbirgt - zugleich aber gibt er Matt Informationen, in der Hoffnung, Daredevil zum Eingreifen zu bewegen (vgl. ebd. 66f.). 
pin (Daredevil) diese negative Konnotierung beiläufig, wenn sie fragen, was der Held wohl zu verbergen hat, wenn er Angst hat, sein (,wahres) Gesicht zu präsentieren. Hier wird deutlich, dass die Maske eben nicht nur verbirgt, sondern auch zeigt, dass sie etwas verhüllt (Weihe 17).

Da die maskierten Helden ihre Superfähigkeiten nur unter der gegenständlichen Maske einsetzen, lässt sich argumentieren, dass ihr, wahres Ich` erst beim Tragen der Maske enthüllt wird, die zivile Identität aber eine metaphorische, soziale Maske bedeutet. Dieses Spiel um die Frage nach dem wahren Ich beschreibt Kniep mit anschließendem Bezug auf Batman und Superman für die Comics folgendermaßen:

Sein wahres Ich ist somit das heldische Ich, das vor dem Hintergrund wechselnder Erscheinungen die Einheit und Dauer eines geschlossenen Subjekts verbürgt. Daraus folgt, dass es nicht der Mensch (Clark Kent, Bruce Wayne usw.) ist, der seine eigentliche Identität hinter der Maske eines Superhelden verbirgt, sondern umgekehrt der Held selbst, der mit Hilfe der Konstruktion einer Alltagspersona unentdeckt zu hoffen bleibt. Der zu der Aufnahme eines Doppellebens dazugehörige Vorgang der Kostümierung stellt sich dem Comic-Leser dann auch folgerichtig als ein paradoxes Wechselspiel zwischen Enthüllung und Verschleierung dar: Legt der Held die Maske ab, bleibt er als solcher unerkannt, setzt er sie aber auf, tritt er als derjenige in Erscheinung, der er in Wahrheit ist. (32f.)

Kniep scheint die ,Umkehrung' von authentischem und vorgegebenem Ich durch die Maskierung als Beantwortung der Frage nach der, wahren Identität' als gegeben anzusehen - Fingeroth (57-60) betont demgegenüber eher, dass die Comics vor allem immer wieder Fragen wie ,Wer bin ich? 'stellen.

Die Umkehr zwischen gegenständlicher und metaphorischer Maske wird in den klassischen Comics durchaus explizit angesprochen. Der Wechsel zwischen den Identitäten bedeutet hier den Wechsel zwischen authentischem und vorgegebenem Ich (z. B. Lee und Everett [Daredevil 17, Juni 1966, 4; Daredevil 25, Februar 1967, 7]). Matt Murdocks Behindertenattribut, sein Blindenstock, der in Daredevils Hand zur Waffe wird, illustriert dies auch im Film. Im Vergleich zu den Comics ist in den Filmen jedoch eine relativ subtile Verschiebung zu beobachten. Obwohl sich der Plot in den ersten beiden Spider-Man-Filmen auch darum dreht, dass sich Peter für eine seiner beiden Identitäten entscheiden muss, fehlt in allen Filmen die Komponente der ,Maskenhaftigkeit' der zivilen Identität. Dass das ZivilIch ein Meister der Verstellung ist und daher manchmal für den Helden nicht mehr als eine zwingende Rolle ist, die er zu erfüllen hat, die seinem Ich aber nicht entspricht, wird so gut wie gar nicht angesprochen. Wenn die Helden hier ihre fantastischen Fähigkeiten vor aller Öffentlichkeit einsetzen, bedeutet die Maskerade daher nicht die ,Umkehrung' von wahrem Selbst und vorgespielter sozialer Rolle, sondern stellt ,nur ${ }^{6}$ eine Erweiterung der Identität dar, ohne aber das ,angestammte' Ich in Frage zu stellen. Aufgrund dieser Umwertung ist es nicht mehr so erstaunlich, dass 
sich die drei Heldenfiguren in ihren Filmversionen nicht übermäßig bemühen, in ihrer zivilen Identität ihre Spezialfähigkeiten nur im Geheimen zu benutzen, um niemandem Anlass zu geben, auf die Verbindung mit der Heldengestalt zu schließen. So entdeckt Peter Parker seine Spinnenkräfte im öffentlichen Raum der Schule, was für ziemlichen Aufruhr sorgt, und der blinde Matt Murdock verblüfft sein Umfeld regelmäßig mit seinen Wahrnehmungsfähigkeiten ${ }^{15}$, bis Elektra direkt fragt: „Sure you're blind?““ (Daredevil 00:35:00).

Diese ,Konstellation“ der Doppelidentität des maskierten Helden bietet sich geradezu an, sie als Widerspiegelung jener Identitätskonzeptionen zu deuten, die Identität als von Multiplizität und Prozesshaftigkeit geprägt $\mathrm{zu}$ verstehen. Diese scheinen in Soziologie und Psychologie weitgehend anerkannt zu sein und die Vorstellung von Identität als einer fixen Entität abgelöst zu haben (vgl. Renn und Straub; Kaufmann). Insbesondere im Blick auf Liftons Protean Self entfalten sich auffällige Parallelen zu den Superhelden, wie sie in den hier betrachteten Filmadaptionen porträtiert werden. Lifton entwirft sein metaphorisch gelungen benanntes Identitätskonzept als vielseitiges Selbst (5), das durch Variation geprägt ist, aber dennoch konstante, stabile Züge hat. Grundlage für die Konstituierung des Protean Self sind unter anderem Gefühle der Vater- und Heimatlosigkeit (ebd. 74). Zumindest Erstere sind bei Peter Parker, Matt Murdock und Tony Stark nicht von der Hand zu weisen. Bedeutender ist jedoch die folgende Eigenschaft: „Central to its function is a capacity for bringing together disparate and seemingly incompatible elements of identity and involvement in [...] 'odd combinations,' and for continuous transformation of these elements" (ebd. 5). Die von Lifton erwähnte ,continuous transformation' kann für die maskierten Helden nicht nachgewiesen werden, sodass die Anwendung des Konzepts des Protean Self hier an ihre Grenzen stößt. Doch lassen sich die beiden Ichs des maskierten Helden wenn auch in sehr plakativer, schematisierter Form - als solch scheinbar inkompatible Elemente verstehen; die Aufrechterhaltung zweier Identitäten ist schließlich eine Grundkompetenz für einen maskierten Helden.

Obgleich die ständige Aufrechterhaltung zweier Identitäten für den maskierten Helden oft kleine und große Probleme nach sich zieht, kann die Doppelidentität auch eine Stärke sein. Es gibt immer wieder Situationen, in denen die Helden keinen Erfolg verbuchen könnten, wenn sie nicht auf die Verbindung zwischen ihren beiden Identitäten und ihre zivile Identität zurückgreifen könnten. In Spider-Man 2 beispielsweise kann angesichts der drohenden Explosion nicht Spider-Man, sondern nur Peter Dr.

15 Zur Umsetzung von Matts spezieller Raumwahrnehmung durch seinen Radarsinn im Film vgl. Kuppers. 
Octopus zur Vernunft bringen und so die Katastrophe abwenden (vgl. Booker 119).

In den klassischen Comics haben Spider-Man, Daredevil und Iron Man jahrelang größte Anstrengungen unternommen, um einer Entlarvung zu entgehen. In den Filmen fällt dagegen eine hohe Zahl an Demaskierungen auf. ${ }^{16}$ Matt Murdock wird im Film insgesamt genauso oft entlarvt, wie er als Daredevil unterwegs ist. Die relative Häufigkeit der Demaskierungen in den Filmen ist in erster Linie sicher darin begründet, dass die Schauspieler ohne Maske ihr Mienenspiel besser entfalten können. Davon abgesehen bedeutet die Demaskierung die Sichtbarmachung der ,Einheit in der Zweiheit ${ }^{617}$ und damit die (temporäre) Aufhebung der vorgegebenen Trennung in zwei Identitäten. Dies kann zusätzliche Gefahr bedeuten, wenn ein Antagonist die Identität des Helden entdeckt und so erkennt, wie er z. B. Spider-Man mit der Bedrohung von Peter Parkers Familie erpressen bzw. in ein Dilemma stürzen kann. Die Maske bietet darüberhinaus im Prinzip nicht nur Schutz vor Superschurken, sondern auch vor der meinungsmäßig oft wankelmütigen Öffentlichkeit, die bei Daredevil und in der Spider-Man-Trilogie stark durch die Presse repräsentiert wird. Eine unfreiwillige Demaskierung vor einem beschränkten Kreis der Öffentlichkeit gibt es nur Spider-Man 2: Peter verliert im Laufe eines Kampfes mit Doctor Octopus vor einigen Stadtbahnbenutzern seine Maske, was jedoch keine negativen Folgen hat - da die New Yorker ihrem Helden dankbar sind, versprechen sie, sein Geheimnis zu wahren. In Liebesdingen führt die Demaskierung dagegen meist zur Problemlösung - so bringt Elektra Daredevil dann doch nicht um, als sie sieht, dass Matt Murdock unter dessen Maske steckt. In den Spider-Man-Filmen ist es eine halb zerrissene Maske, die vor allem in den Endkämpfen der drei Spider-Man-Filme wenn Peter Parker als Spider-Man seine große Liebe Mary Jane retten muss - sichtbar macht, dass Spider-Man Peter Parker ist, und immer dann, wenn die Doppelidentität bei der Kulmination handlungsbedeutsam ist, die Verbindung der beiden Identitäten eindrücklich visualisiert. Dies ist freilich auch eine Hommage an ein typisches Gestaltungselement der Spider-Man-Comics, dort ,enthüllt ${ }^{\star}$ eine über Peters Gesicht gezeichnete Halbmaske, dass Peter gerade als Spider-Man denkt (vgl. etwa Lee und Ditko [z. B. The Amazing Spider-Man 4, September 1963, 20; 5, Oktober 1963, $2,10,11])$.

16 Iron Man, der am Ende die unumkehrbare Demaskierung gegenüber der Öffentlichkeit vornimmt, kann von Demaskierungen ,den Zuschauenden zuliebe‘ absehen, da in einer speziellen Kameraeinstellung Tony Starks Gesicht hinter der Maske mehrmals gezeigt wird.

17 Diese Formulierung verwende ich in Anlehnung an Weihes Auseinandersetzung mit der Denkfigur des homo duplex, deren Bezügen zum maskierten Helden hier nicht nachgegangen werden kann (vgl. 329-54). 


\section{Wer die Wahl (nicht) hat ...}

Anders als bei einer Demaskierung, die in den Filmen in der Regel unfreiwillig oder zumindest ungeplant erfolgt, können Spider-Man und Daredevil in der Regel selbst bestimmen, wann sie in welcher Identität auftreten wollen (das gilt auch für Iron Man, solange er nicht in seiner Rüstung feststeckt). Die äußerliche Trennung in zwei Identitäten stellt die Protagonisten so in gewisser Weise dauernd vor die Wahl, da sie gegen außen jeweils nur in einer ihrer Identitäten auftreten können, nicht aber in beiden gleichzeitig.

Auch eine Doppelidentität ist keineswegs vor Krisen gefeit. Immer wieder ein effektvolles Mittel, den Protagonisten in ein Dilemma zu stürzen, ist, ihn vor die sprichwörtliche Qual der Wahl zu stellen, sich für eine seiner beiden Identitäten entscheiden zu müssen, wie Spider-Man und Spider-Man 2 zeigen. Im ersten Film stellt der Green Goblin Spider-Man vor das Dilemma, entweder Mary Jane oder eine Gondel voller Schulkinder zu retten. Mit den Worten „We are who we choose to be. Now choose!“" (01: 38:02), fordert er Spider-Man auf, sich dergestalt zwischen seinen Identitäten zu entscheiden. Selbstverständlich gelingt es Peter, sowohl die Frau seines Herzens als auch die Schulkinder zu retten. Er beschließt im Anschluss jedoch, wegen der potentiellen Gefahren auf eine Beziehung mit Mary Jane zu verzichten, um Spider-Man bleiben zu können. Das finale „Who am I? I am Spider-Man“ (01:50:33) lässt sich dennoch nicht nur als Bekenntnis zum Heldentum lesen, sondern auch als Entscheidung zur Erhaltung der Doppelidentität. ${ }^{18}$ Und damit startet denn auch der zweite Film, wenn der erste Satz von Peters Erzählerstimme lautet: ,Who am I? I am Spider-Man, given a job to do. And I'm Peter Parker, and I, too, have a job" (00:03:10).

Nach diesem Statement ist es unvermeidlich, dass die Doppelidentität im zweiten Film in die Krise gerät, bis Peter entnervt seine Kräfte nicht mehr abrufen kann und den Helden-Bettel hinschmeißt, was scheinbar den Verzicht auf sein heldisches Alter Ego bedeutet. Da er jedoch seine Heldenqualitäten nicht begraben kann (wie sich zeigt, ist er nicht aus dem Holz geschnitzt, das bei Unglücken seelenruhig zuschauen kann) und diese auch dringend gebraucht werden, als der Superschurke Dr. Octopus seine große Liebe Mary Jane entführt, wird die Doppelidentität am Ende, wie zu erwarten war, wieder bestätigt. Beim Happy-End wird deutlich, dass die Ausgestaltung der Identität auch vom sozialen Umfeld und den Interaktionen mit anderen abhängig ist: Peter zieht sich nämlich erneut

18 Laut Kirby und Gaithner (280) unterscheidet sich Peter Parker in Spider-Man in diesem Punkt als relativ singuläres Beispiel von anderen, etwa zur gleichen Zeit produzierten Filmen, in denen Individuen durch genetische Experimente vor die Identitätsfrage gestellt werden. 
von Mary Jane zurück, da diese aber inzwischen sein Geheimnis kennt, entscheidet sie, dass sie trotzdem mit Peter zusammen sein will.

Flanagan weist darauf hin, dass die Doppelidentität dem Helden (und implizit dem Kinopublikum, das sich mit diesem identifiziert) die Möglichkeit gibt, verschiedene Rollen - er nennt jene des Verfolgten, des Retters und der Bedrohung - erfüllen zu können. Dieses Potenzial erkläre zum einen die Langlebigkeit der Secret Identity und diene zum andern der Rechtfertigung verschiedener ,narratives that deal with the assumption of new identities, heroic guises, or the temporary rejection of the hero identity" (145). Dementsprechend kann Peters Identität in Spider-Man 3 ebenfalls nicht unhinterfragt bleiben, die Doppelidentität als solche steht nun aber nicht mehr zur Debatte. Insbesondere als 'Spider-Man in Black' unter dem Einfluss des außerirdischen Symbionten - kann Peter Parker eine vermeintlich coolere, selbstsicherere und frechere Variante seiner selbst testen, die er zunächst begrüßt, letztlich aber wieder verwerfen muss, als er erkennt, dass dieses eine gnadenlose, gewalttätige Dimension an den Tag legt, die ihm nicht geheuer ist. Peters Fazit am Schluss von Spider-Man 3 betont erneut, mit schon fast didaktischem Unterton und in fast denselben Worten wie der Goblin im ersten Teil, die Wahl: „We always have a choice. [...] It's the choices that make us who we are. And we can always choose to do what's right" (02:06:25).

Der Aspekt der Wahl des Protagonisten, wer er sein will, wird vor allem in der Spider-Man-Trilogie betont. Die starke Gewichtung lässt sich dadurch erklären, dass Peter Parkers Transformation zu Spider-Man - er erhält seine Spinnenkräfte noch als Teenager - oft als Metapher für die Entwicklungen in der Pubertät gelesen wird (z. B. Flanagan 139-50; Booker 109). Dementsprechend versteht etwa Flanagan das Dilemma der Wahl zwischen zwei Identitäten in Spider-Man hauptsächlich als Form, in die das Problem der Heldwerdung bzw. der Akzeptanz des Heldenschicksals (,rising to a heroic destiny“ 148) erzählerisch eingekleidet ist. Mit dem drohenden Verzicht auf eine der beiden Identitäten geht es jeweils auch um die Frage, ob Peter ein Held mit aller Verantwortung und Entbehrungen für seine Zivilidentität sein will oder nicht. Daran schließt sich - namentlich in Gesprächen von Peter Parker mit seiner nicht eingeweihten Tante May über Spider-Man - die Frage an, was einen Helden und seine Vorbildfunktion ausmacht. ${ }^{19}$ Aber während ein Leben ohne Heldenidentität durchaus denkbar wäre - was in Spider-Man 2 temporär auch durchgespielt wird, als Peter seine Spinnenkräfte verliert -, scheint die Beibehal-

19 Im Film Daredevil schwingt die ,Heldenfrage‘ in einer anderen Schattierung mit, hier befindet sich Matt im Zwiespalt, ob der stark vergeltungsorientierte Daredevil noch ein Held ist oder ob er zu weit geht und so selbst zum Problem wird - er kann dieser ,Versuchung aber widerstehen und bleibt selbst ernannter ,guardian devil“ (01:32:38) seines Viertels. 
tung der zivilen Identität des Protagonisten unumstößlich. Auch wenn das zivile Ich durch die Heldenaktivitäten eingeschränkt wird (z. B. wenn Peter Parker auf privates Liebesglück verzichtet), scheint eine reine Heldenidentität keine Option.

Die Entscheidung, wer er sein will, muss Peter Parker in den SpiderMan-Sequels immer wieder neu treffen. Das Fantastische, das die Doppelidentität erst ermöglicht hat, dient dabei meist als Anstoß, die gewählte Identität zu hinterfragen. Namentlich in Form des außerirdischen Symbionten im dritten Teil zwingt es Peter dazu, eine andere Schattierung seiner Identität auszuprobieren, wobei Peter schließlich, wenn auch in einem Kraftakt, die etablierte Doppelidentität bestätigt.

Abschließend sei ein kurzer Blick auf die Superschurken der SpiderMan-Filme geworfen, denn in Bezug auf die Wahlmöglichkeit zwischen den Identitäten ergibt sich ein wichtiger Unterschied zu den Superhelden. Wie erwähnt, verfügen Superschurken über vergleichbare Origin Storys wie die Helden (meistens erhalten sie ebenfalls durch einen Unfall in wissenschaftlichem Umfeld Superkräfte). Doch haben sowohl Norman Osborn alias Green Goblin ${ }^{20}$ als auch Otto Octavius alias Doctor Octopus keine Wahl, wann sie was (respektive wer) sind, da es der Wahnsinn ist, der ihre Sinne vernebelt. In gewissem Sinn gilt das auch für Venom und Sandman in Spider-Man 3: Da Venom von dem außerirdischen Symbionten in Besitz genommen wurde, ist nicht klar, wie frei er seine Entscheidungen trifft. Der Sandman könnte theoretisch zwar wählen, da er bei Verstand ist, doch weil ihm als Kriminellen eine Rückkehr zu seiner Familie verwehrt wird, verfügt er nicht wirklich über eine zivile Identität, in die er sich zurückziehen könnte. Harry Osborn als New Green Goblin in Spider-Man 3 ist der einzige Superschurke, der in diesem Punkt eine Wahl hat, die auch eine ist; er trifft zuerst die falsche, korrigiert sie aber und darf bzw. muss schließlich den Heldentod sterben.

Die Doppelidentität von Spider-Man, Daredevil und Iron Man (soweit sich das für letzteren filmischen Spezialfall bestimmen lässt) zeichnet sich dagegen in den hier betrachteten Filmen dadurch aus, dass frei zwischen zwei durchaus kontrastreichen, äußerlich getrennten Identitäten hin- und hergewechselt werden kann. Die Doppelidentität vereinigt also ständigen Wechsel, auf der einen Seite und Konstanz auf der andern Seite; denn falls sie einmal etabliert ist, bleibt die Doppelidentität als solche letztlich (bei Filmende) stets intakt, was außertextuell im seriellen Charakter dieser Heldenreihen begründet ist.

20 Laut Flanagan, der sich auf den Green Goblin in Spider-Man bezieht, macht dieser Umstand deutlich, dass die emotionale Reife, mit der die Rolle eines Helden zu erfüllen sei, nicht von der physischen Veränderung durch den Erhalt der Kräfte abhängig ist (156). Zur ,gespaltenen Identität‘ des Green Goblin vgl. Meyer. 


\section{Literaturverzeichnis}

Booker, M. Keith. „The Spider-Man Film Franchise“. May Contain Graphic Material: Comic Books, Graphic Novels, and Film. Wesport: Praeger, 2007. 109-23.

Coogan, Peter. Superhero: The Secret Origin of a Genre. Austin: MonkeyBrainBooks 2006.

Fingeroth, Danny. Superman on the Couch: What Superheroes Really Tell Us about Ourselves and Our Society. New York: Continuum, 2004.

Flanagan, Martin. „Teen Trajectories in Spider-Man and Ghostworld“. Film and Comic Books. Hg. Ian Gordon, Mark Jancovich und Matthew P. McAllister. Mississippi: UP of Mississippi, 2007. 137-59.

Kaufmann, Jean-Claude. Die Erfindung des Ich: Eine Theorie der Identität. Konstanz: UVK 2004.

Kirby, David A. und Laura A. Gaithner. „Genetic Coming of Age. Genomics, Enhancement, and Identity in Film“. New Literary History 36.2 (2005): 263-82.

Kniep, Mathias. Die drei Zeitalter des Superhelden-Comics (Gold, Silber und Bronze): Von der Geburt, Demontage und Wiederbelebung eines amerikanischen $M y$ thos. Kiel: Ludwig, 2009.

Koh, Wilson. „Everything Old is Good Again: Myth and Nostalgia in Spider-Man“. Continuum 23.5 (2009): 735-47.

Kuppers, Petra. „Blindness and Affect: Daredevil’s Site/Sight“. Quarterly Review of Film and Video 23 (2006): 89-96.

Lee, Stan, Don Heck et al. Essential Iron Man. Bd. 1: Tales of Suspense 3972 [1963-1965]. New York: Marvel Comics, 2005.

Lee, Stan, Bill Everett et al. Essential Daredevil. Bd 1: Daredevil 1-25 [196467]. New York: Marvel Comics, 2005.

Lee, Stan, Steve Ditko et al. Essential Spider-Man. Bd. 1: Amazing SpiderMan 1-20, Annual 1 [1962-65]. New York: Marvel Comics, 2002.

Lifton, Robert Jay. The Protean Self: Human Resilience in an Age of Fragmentation. New York: BasicBooks, 1993.

Meyer, Michaela D. E. „Utilizing Mythic Criticism in Contemporary Narrative Culture: Examining the 'Present-Absence' of Shadow Archetypes in Spider-Man“. Communication Quarterly 51.4 (2003): 518-29.

Peaslee, Robert M. „Superheroes, 'Moral Economy' and the 'Iron Cage': Morality, Alienation, and the Super-Individual". Super/Heros: From Hercules to Superman. Hg. Wendy Haslem, Angela Ndalianis und Chris Mackie. Washington: New Academia, 2007. 37-50.

Rauscher, Andreas. „Stadtneurotiker, Outlaws und Mutanten: Das MarvelUniversum im Film“. Superhelden zwischen Comic und Film. Hg. Andreas Rauscher und Andreas Friedrich. München: text+kritik, 2007. 51-71. 
Renn, Joachim und Jürgen Straub. „Transitorische Identität: Der Prozesscharakter moderner personaler Selbstverhältnisse“. Transitorische Identität. Hg. Joachim Renn und Jürgen Straub. Frankfurt/M.: Campus, 2002. 10-31.

Spiegel, Simon. Die Konstitution des Wunderbaren: Zu einer Poetik des ScienceFiction-Films. Marburg: Schüren, 2007.

Tseëlon, Efrat. „Introduction: masquerade and identities“. Masquerade and Identities: Essays on gender, sexuality and marginality. Hg. Efrat Tseëlon. London: Routledge, 2001. 1-17.

Tyree, J. M. „American Heroes: On Frivolity and Horror in 2008's Summer Superhero Movies: The Dark Knight, The Incredible Hulk, and Iron Man." Film Quarterly 62.3 (2009): 28-34.

Weihe, Richard. Die Paradoxie der Maske: Geschicbte einer Form. München: Fink, 2004.

Filme

Daredevil. Reg. Mark Steven Johnson. Darst. Ben Affleck, Jennifer Garner. DVD. Zürich: PlazaVista, 2003.

Iron Man. Reg. Jon Favreau. Darst. Robert Downey Jr., Gwyneth Paltrow. 2008. DVD. München: Concorde, 2009.

Iron Man 2. Reg. Jon Favreau. Darst. Robert Downey Jr., Gwyneth Paltrow. 2010. Film.

Spider-Man. Reg. Sam Raimi. Darst. Tobey Maguire, Kirsten Dunst. 2002. DVD. München: Columbia Tristar, o. J.

Spider-Man 2. Reg. Sam Raimi. Darst. Tobey Maguire, Kirsten Dunst. 2004. DVD. München: Columbia Tristar, 2004.

Spider-Man 3. Reg. Sam Raimi. Darst. Tobey Maguire, Kirsten Dunst. 2007. DVD. München: Sony, 2007. 